\title{
La importancia de la segregación de itrio en la deformación plástica de nanocristales de circonia tetragonal dopada con óxido de itrio (YTZP)
}

\author{
C. LORENZO MARTíN, A. GALLARDO LÓPEZ, D. GÓMEZ GARCíA Y A. DOMÍNGUEZ RODRÍGUEZ. \\ Centro de Investigación y Departamento de Física de la Materia Condensada, Universidad de Sevilla. Apdo. 1065, 4180, Sevilla.
}

\begin{abstract}
Muestras de YTZP (policristales de oxido de circonio dopado con itria) con tamaño medio de grano de $50 \mathrm{~nm}$, puras y con un $10 \%$ en peso de diferentes fases vítreas han sido deformadas a carga constante en un rango de temperatura comprendido entre 1150-1200 ${ }^{\circ} \mathrm{C}$. Con dicho estudio se pretende caracterizar el comportamiento mecánico del material y estudiar la influencia de la segregación de itria en la frontera de los granos en los posibles mecanismos de deformación. Igualmente se ha llevado a cabo un estudio microscópico con el fin de obtener una completa caracterización del material. Se han analizado micrografías de las muestras recibidas y de las deformadas a partir de Microscopía Electrónica de Transmisión convencional (TEM), y de las fronteras de granos de dichas muestras por Microscopía Electrónica de Transmisión de Alta Resolución (HRTEM).

Se ha probado que la existencia de segregación catiónica hacia las fronteras de granos está relacionada con la falta de una tensión umbral. El parámetro n no se ve alterado por dicha segregación, en buen acuerdo con la ausencia de tensión umbral.
\end{abstract}

Palabras claves: Nanocerámicos, YTZP, fase vitrea, segregación.

Importance of yttrium segregation on plastic deformation of nanocrystalline ytzia doped tetragonel zirconia (YTZP)

Nano-sized YTZP (Yttria Tetragonal Zirconia Polycrystals) samples, pure and doped with 10 wt.\% of different glassy phases have been crept in a range of temperature between 1150-1200 ${ }^{\circ} \mathrm{C}$. Macroscopic characterization of the mechanical behaviour of these material and a study on the effect of the grain boundaries yttria segregation on the mechanical response of these materials have been carried out. Moreover, a microstructural analysis by conventional Transmission Electron Microscopy (TEM) of both, as-received and deformed samples and a study of the grain boundaries by High Resolution Transmission Electron Microscopy are required to have a complete characterization of these materials.

The yttrium segregation to grain boundary is related to the non existence of a threshold stress. The parameter $\mathrm{n}$ is not altered by this segregation and grain boundary sliding seems to be the deformation mechanism.

Key words: Nanoceramics, YTZP, glassy phase, segregation.

\section{INTRODUCCIÓN}

La ciencia de los nanomateriales es hoy día una de las líneas de investigación de más rápido crecimiento. Dentro de ella, los materiales cerámicos nanoestructurados despiertan un enorme interés, ya que pueden presentar propiedades muy diferentes a los materiales cerámicos convencionales, abriéndose así la posibilidad de diseño de materiales cerámicos con potenciales aplicaciones que los haga competitivos frente a los metales, tal como el "conformado superplástico" y uniones de materiales cerámicos (1). Los materiales cerámicos a base de circonia constituyen la vanguardia en este campo debido a la superplasticidad exhibida en el rango submicrométrico (2). Aunque es escasa la literatura existente para estos materiales cerámicos nanométricos, un primer análisis ha sido el estudio realizado por Gutiérrez Mora y col. (3) y por Lorenzo Martín y col. (4) acerca del comportamiento mecánico a alta temperatura de los mismos. De ellos se desprende que el deslizamiento de fronteras de granos es el mecanismo principal de deformación, aunque no se concluye cuál es el mecanismo de acomodación. También se estudia el papel jugado por la existencia de la fase vítrea, que se traduce en un efecto de ablandamiento del material (5). En este trabajo vamos a centrarnos en estudiar la existencia de segregación de itria, así como el papel que desempeña en la respuesta mecánica del material.

\section{2.-PROCEDIMIENTO EXPERIMENTAL}

\section{1.- Ensayos de fluencia}

Se dispone de muestras de materiales cerámicos nanométricos de YTZP sin dopar (que en lo que sigue llamaremos puras) y dopadas con un $10 \%$ de distintas fases vítreas. El análisis químico del material recibido revela que la composición química de dichas fases vítreas es de un $80 \%$ en peso de $\mathrm{SiO}_{2}, 15 \%$ en peso de $\mathrm{Al}_{2} \mathrm{O}_{3}$, y $5 \%$ en peso de $\mathrm{SrO}$, para la denominada "SAS" y un $80 \%$ en peso de $\mathrm{SiO}_{2}, 15 \%$ en peso de $\mathrm{Na}_{2} \mathrm{O}$, y $5 \%$ en peso de $\mathrm{SrO}$ para la que llamaremos "SNS". Las densidades experimentales están todas por encima del $97 \%$ del valor teórico medido con relación a las muestras puras $\left(6.1{\mathrm{gr} . \mathrm{cm}^{-3}}^{-3}\right.$.

Las muestras fueron cortadas en forma de paralelepípedo y dispuestas entre pistones de alúmina, interponiendo pastillas de carburo de silicio entre los pistones y la muestra con el fin de evitar posibles indentaciones. Los ensayos de deformación a carga constante en compresión (fluencia), en compresión se realizaron utilizando una máquina prototipo descrita en (6), en un rango de temperatura entre 1150 y $1210{ }^{\circ} \mathrm{C}$, con tensiones comprendidas entre 5 y $35 \mathrm{MPa}$, y alcanzando 
velocidades de deformación comprendidas entre $10^{-7}$ y $10^{-5}$ $\mathrm{s}^{-1}$. La temperatura de trabajo es suficientemente baja para que no exista crecimiento dinámico de los granos pero a la vez suficientemente alta para que la difusión juegue un papel relevante en el proceso de deformación.

\section{2.- Observaciones microestructurales y microanálisis}

Tras la deformación se observó las microestructura de las muestras y se comparó con el material sin deformar, con el fin de observar si existe evolución microestructural durante el ensayo de deformación. Como se verá posteriormente, se trata de un dato muy relevante para determinar el mecanismo de deformación. Para ello se prepararon muestras para ser observadas por TEM y HRTEM siguiendo el procedimiento convencional descrito en (4). Las muestras fueron estudiadas mediante TEM utilizando un microscopio (Philips CM200) operando a $200 \mathrm{kV}$, del Servicio de Microscopía de la Universidad de Sevilla. Posteriormente fueron analizadas químicamente, obteniéndose perfiles de concentración de ciertas especies a lo largo de fronteras de granos mediante la técnica de espectroscopía de rayos $\mathrm{X}$ emitidos por excitación electrónica (XEDS), utilizándose para ello un microscopio análogo (Philips CM200) convenientemente equipado. Las fronteras de los granos fueron observadas mediante HRTEM empleando un microscopio de resolución atómica ARM-1000 JEOL, operando a $800 \mathrm{kV}$; ambos microscopios pertenecientes al Servicio de Microscopía Electrónica (N.C.E.M) del Laboratorio "Lawrence Berkeley" de la Universidad de Californía. Para estimar el tamaño medio de grano, se obtuvieron diagramas de rayos $\mathrm{X}$ de las distintas muestras, empleando para ello un difractómetro Philips PW-1700 de la Universidad de Extremadura.

\section{RESULTADOS}

Los datos que se obtuvieron de los ensayos de fluencia se ajustaron a la ecuación constitutiva de fluencia a altas temperaturas:

$$
\dot{\varepsilon}=A \frac{G b}{k T}\left(\frac{\sigma-\sigma_{0}}{G}\right)^{n} D_{0} \exp \left(\frac{-Q}{k T}\right)
$$

siendo $\mathrm{E}$, la velocidad de deformación; A, una constante no dimensional; $\mathrm{G}$, el módulo de cizalladura; $b$, el vector de Burgers de las dislocaciones en YTZP; $\sigma$, la tensión aplicada; T, la temperatura absoluta; k, la constante de Boltzmann y $\mathrm{D}_{0}$ un factor preexponencial del coeficiente de difusión. El término $\sigma_{0^{\prime}}$ conocido como tensión umbral, es la mínima tensión necesaria para que exista deslizamiento de los granos y es una función inversa del tamaño de grano (7). Los parámetros $\mathrm{n}$ (exponente de tensión) y Q (energía de activación) se determinan experimentalmente mediante cambios de la tensión o de la temperatura, manteniendo los otros parámetros constantes (figura 1) y son característicos del proceso de deformación del material. El cálculo de $\mathrm{n}$ se hace mediante la siguiente expresión:

$$
n=\frac{\operatorname{Ln}\left(\dot{\varepsilon_{2}} / \dot{\varepsilon}_{1}\right)}{\operatorname{Ln}\left(\sigma_{2} / \sigma_{1}\right)}
$$

siendo $E_{1}$ y $E_{2}$ las velocidades de deformación estacionarias antes del salto $\left(\sigma_{1}\right)$ y después del salto $\left(\sigma_{2}\right)$; ambas medidas para un mismo valor de la temperatura y la deformación. De forma análoga se procede para calcular el parámetro $Q$, a partir de tramos con diferentes temperaturas en donde las velocidades de deformación están calculadas en las mismas condiciones de deformación y tensión.

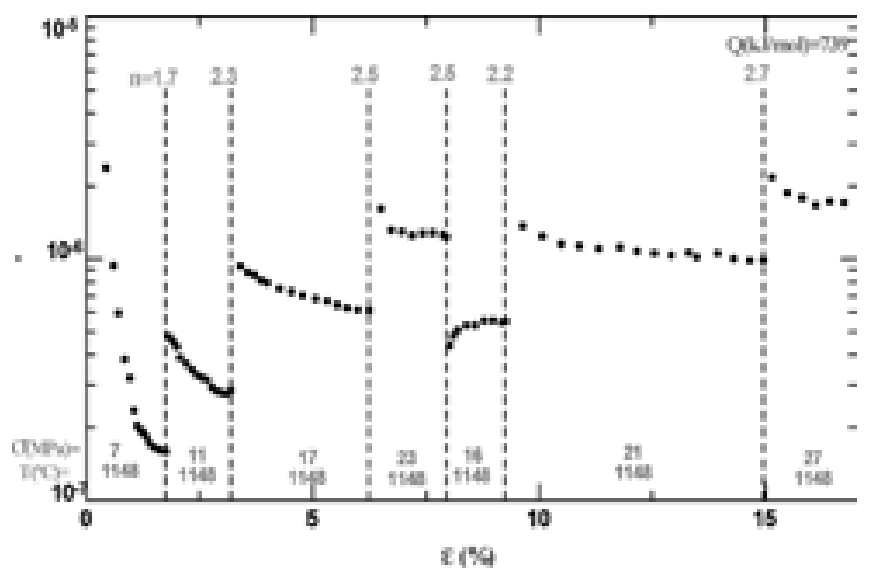

Fig. 1.- Curva de fluencia para la muestra con un $10 \%$ de fase vítrea tipo SNS. Se muestran los valores obtenidos para el exponente de tensión (n) y la energía de activación (Q) para las distintas condiciones de ensayo.

En la figura 1 puede verse varios valores de $n$ y $Q$ junto con las condiciones experimentales utilizadas para su cálculo. El valor medio de los resultados obtenidos para $n$ y $Q$ en los distintos tipos de muestras estudiadas se recogen en la tabla I.

TABLA I.- VALORES DEL EXPONENTE DE TENSIÓN Y LA ENERGÍA DE ACTIVACIÓN CALCULADOS A PARTIR DE LAS EXPERIENCIAS DE FLUENCIA

\begin{tabular}{|c|c|c|}
\hline muestra & $\mathbf{n}$ & $\mathbf{Q}(\mathbf{k J} / \mathbf{m o l})$ \\
\hline Pura & $2.0 \pm 0.4$ & $682 \pm 15$ \\
\hline $10 \%$ fase vítrea SNS & $2.3 \pm 0.5$ & $710 \pm 40$ \\
\hline $10 \%$ fase vítrea SAS & $2.3 \pm 0.5$ & $710 \pm 30$ \\
\hline
\end{tabular}

Puede comprobarse que, si bien los valores obtenidos para el exponente de tensión están en buen acuerdo con los obtenidos para el mismo material con tamaño medio de grano en el rango submicrométrico $(n=2)(8)$, el valor obtenido para la energía de activación al disminuir el tamaño medio de grano a las decenas de nanómetros es bastante superior $(\mathrm{Q} \cong 700 \mathrm{~kJ} / \mathrm{mol}$ ) frente al valor obtenido para las muestras submicrométricas $(Q \cong 500 \mathrm{~kJ} / \mathrm{mol})$. Esto ocurre tanto en la muestra pura como en las que contiene fase vítrea.

Para estudiar la posible evolución microestructural del material durante las experiencias de deformación, se han observado las muestras por TEM del material antes y después de ser deformado (figura 2). Cabe destacar que tanto el tamaño como la forma de los granos se mantienen constantes, y no se observan actividad de dislocaciones, cavitación ni grietas. Además, este hecho es independiente de la cantidad 

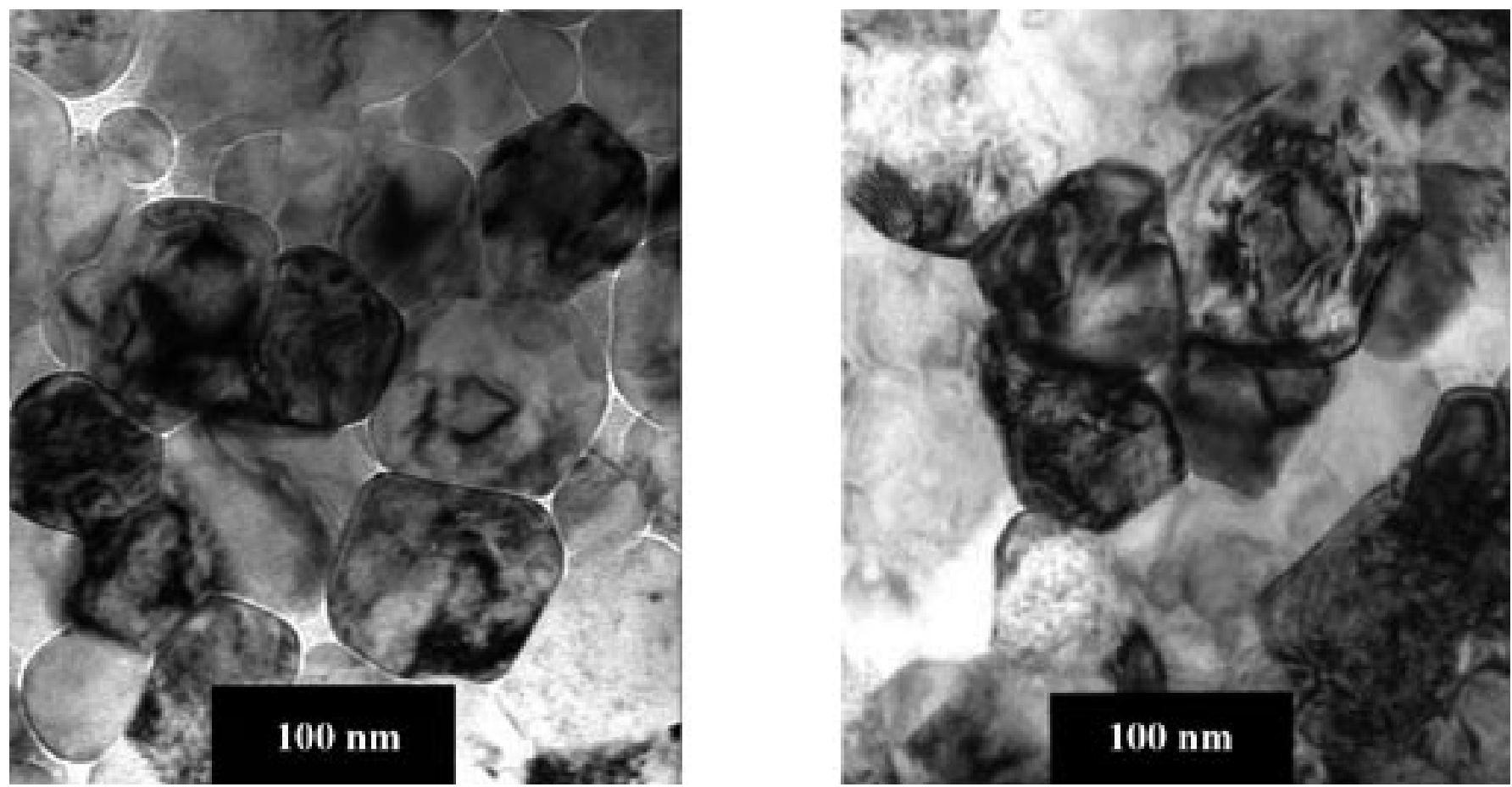

Fig. 2.- Micrografías de la muestras $10 \%$ con fase vítrea SAS, antes de ser deformada (derecha) y después de ser deformada (izquierda)

de impurezas que contiene el material. Por otro lado, para la estimación del tamaño medio de grano, los diagramas de rayos $X$ obtenidos fueron analizados mediante la técnica de Warren-Aberbach (9). Con este método se obtiene una cota inferior del tamaño medio de grano, que no difiere mucho del valor real para distribuciones estrechas. El tamaño medio de grano, calculado mediante esta técnica está en torno a $50 \pm 20$ $\mathrm{nm}$ para todas las muestras. En la figura 3 se muestra el histograma obtenido para la muestra con un 10\% de fase vítrea SAS. Los valores de tamaños de granos así estimados están en buen acuerdo con los obtenidos para los mismos materiales mediante microscopía de fuerza atómica (AFM) (4).

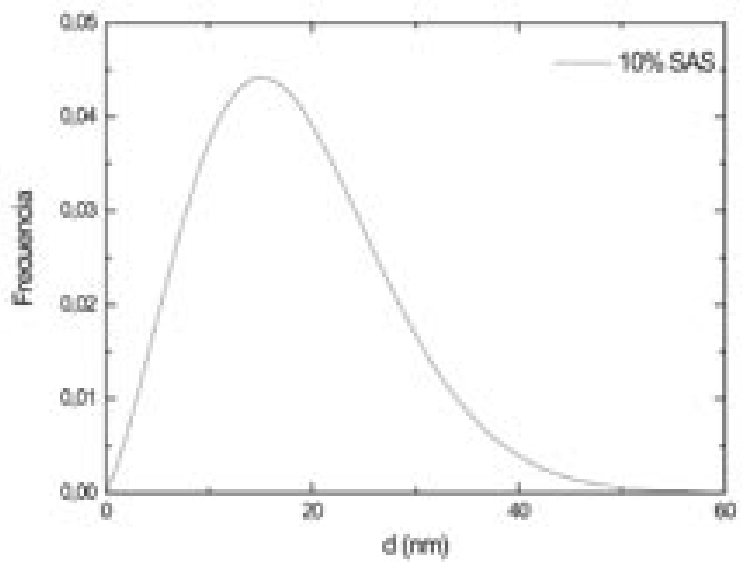

Fig. 3.- Distribución log-normal del tamaño de grano para la muestra $10 \%$ SAS

Con el objeto de establecer si existe segregación de itrio en las fronteras de los granos y analizar las implicaciones que conlleva dicha segregación, se llevó a cabo un microanálisis de las muestras de estudio mediante XEDS. En todas las muestras estudiadas se encontró un incremento de la concentración de itrio en torno al borde de los granos, resultando ser inferior al $1 \mathrm{~mol} \%$.

Por último, el análisis de las muestras mediante HRTEM revela que existe una acumulación de fase vítrea en los puntos triples, disminuyendo fuertemente a medida que nos aproximamos al punto de contacto entre granos en donde deja de ser distinguible (figura 4).

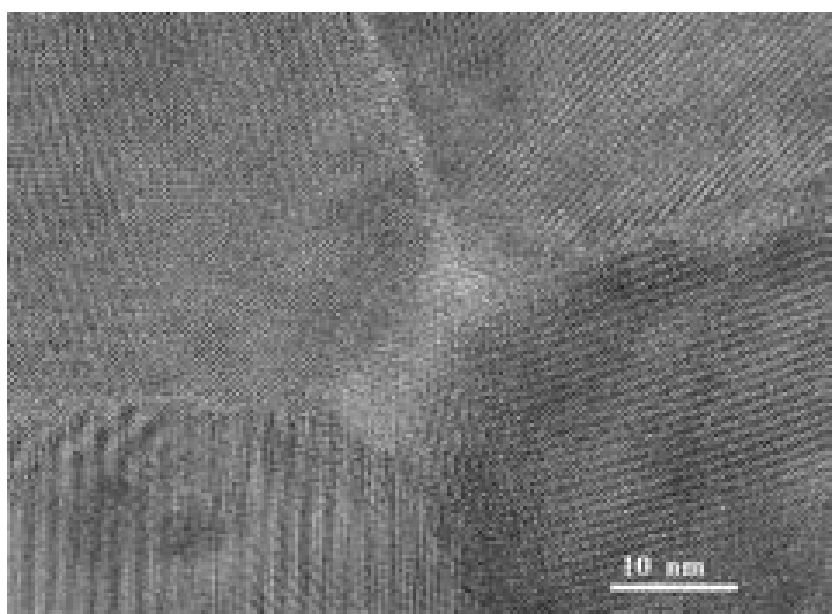

Fig. 4.- Micrografía de transmisión de una unión de fronteras de granos para la muestra con un $10 \%$ de fase vítrea SAS

\section{DISCUSIÓN}

Los valores obtenidos para los parámetros característicos de la fluencia, en concreto el valor en torno a $n=2$ para el exponente de tensión, y el hecho de que no exista cambio microestructural del material durante la deformación, 
manteniéndose constante tanto la forma como el tamaño medio de los granos, indica que el principal mecanismo de deformación presente bajo estas condiciones de ensayo es el deslizamiento de fronteras de granos. Este mecanismo de deformación propuesto está de acuerdo con la amplia literatura existente para este material en el rango submicrométrico (7) y con los estudios preliminares ensayados para muestras con tamaño de grano en el rango nanométrico $(3,4)$. Con respecto a la energía de activación, el valor obtenido para nuestro material de estudio $(Q \cong 700 \mathrm{~kJ} / \mathrm{mol})$, resulta ser muy superior al valor de la energía de activación cuando el sistema acomoda por difusión catiónica en volumen $(Q \cong 500 \mathrm{~kJ} / \mathrm{mol})$, mecanismo de acomodación aceptado para las muestras en el rango submicrométrico. Aunque este resultado también está de acuerdo con los datos obtenidos en incipientes estudios desarrollados para nuestro material $(3,4)$, no se conocen las causas que hacen aumentar este parámetro, quedando aún por resolver cuál es el mecanismo de acomodación presente.

Otra de las diferencias importantes con las muestras en el rango submicrométrico es la no existencia de tensión umbral en nuestro rango de trabajo. De los ensayos de fluencia realizados se desprende que en el caso de existir tensión umbral para las muestras ensayadas, ésta sería inferior a $\sigma_{0}$ $\cong 5 \mathrm{MPa}$ (figura 1). Sin embargo, la tensión umbral predicha para estos nanocerámicos, con un tamaño medio de grano en torno a los $50 \mathrm{~nm}$ y a una temperatura de trabajo de 1150 ${ }^{\circ} \mathrm{C}$, de acuerdo al comportamiento del material en el rango submicrométrico, sería de $160 \mathrm{MPa}$ (8). Vemos por tanto que al disminuir el tamaño de grano, la dependencia $1 / \mathrm{d}$ de la tensión umbral deja de tener sentido.

Un modelo general que recoge dicho comportamiento ha sido recientemente propuesto por Gómez-García y col. $(10,11)$. En éste se apunta a que la diferencia de comportamiento encontrada en ambos rangos, está relacionada con la disminución de segregación de las especies catiónicas en la frontera de grano conforme se disminuye el tamaño de grano hasta las decenas de nanómetros. Dicha segregación posibilita la formación de campos eléctricos locales capaces de alterar el transporte de materia de especies cargadas. En el caso de materiales metálicos existe una compensación perfecta de carga debida a la alta movilidad de los electrones, mientras que en materiales cerámicos la compensación no es total y existe una zona restringida, de unos nanómetros en torno al borde del grano, en la que el campo eléctrico es no nulo. Cuando la segregación es débil, los efectos del campo eléctrico se minimizan de forma que la fuerza electrostática que se opone al movimiento relativo de los granos es despreciable. Este efecto sería el responsable de la baja tensión umbral presente en muestras nanométricas (12)

\section{CONCLUSIONES}

Los ensayos de fluencia con muestras de YTZP con tamaño medio de grano de $50 \mathrm{~nm}$ y en un rango de temperatura entre $1150-1200{ }^{\circ} \mathrm{C}$, junto a un análisis microscópico de las muestras antes y después de ser deformado, han revelado que el material se deforma mediante deslizamiento de fronteras de grano siendo aún objeto de controversia el mecanismo de acomodación que tiene lugar en estos materiales. Un análisis químico de las muestras ensayadas indica la existencia de una débil segregación de itrio hacia las fronteras de los granos. Dicha segregación es la responsable de la ausencia de tensión umbral, que se ha obtenido experimentalmente para el material objeto de estudio, según un modelo propuesto recientemente.

\section{AGRADECIMIENTOS}

Los autores agradecen la ayuda financiera aportada por la "Comisión Interministerial de Ciencia y Tecnología" a través del proyecto MAT2000-0622, y MAT2003-04199-CO2-02.

\section{BIBLIOGRAFÍA}

1.- A. Domínguez-Rodríguez, F. Gutiérrez-Mora, M. Jiménez-Melendo and R. Chaim, "Current understanding of superplastic deformation of Y-TZP and its application to joining", Mater. Sci. Eng. A302 154-161 (2001).

2.- F. Wakai, S. Sakagushi and Y. Matsuno, "Superplasticity of Yttria-Stabilized Tetragonal ZrO2 polycrystals", Adv. Ceram. Mater. 1 259-263 (1986).

3.- F. Gutiérrez-Mora, M. Jiménez-Melendo, A. Domínguez-Rodríguez, R. Chaim, "High temperature mechanical behavior of YSZ nanocrystals"; Key Eng. Mat; 787, 171-174 (2000).

4.-C. Lorenzo-Martín, J.A. Flores-Vazquez , D. Gómez-García, A. MuñozBernabé, A. Domínguez-Rodríguez , D. Xue Ming, J. Gómez-Herrero, "Mechanical Behaviour of YTZP nanocrystalline ceramics as a function of the glassy-phase content", J. Eur. Ceram. Soc; 22 14-15 2603-2607 (2002).

5.- C. Lorenzo-Martín, D. Gómez-García, A. Muñoz-Bernabé, A. DomínguezRodríguez. "Fluencia a alta temperatura de policristales con tamaño de grano nanométrico de YTZP dopados con diferentes cantidades de fase vítrea", Bol. Soc. Esp. Ceram. V; 43 [2] 521-523 (2004).

6.- H. Gervais, B. Pellicier and J. Castaing, "Machine de fluage pour essais en compression a hautes temperatures de matériaux céramiques", Rev. Int. Htes. Temp. et Réfract; 15 43-47 (1978).

7.- M. Jiménez-Melendo, A. Domínguez-Rodríguez, A. Bravo-Leon “ Superplastic flow of fine-grained yttria-stabilized zirconia polycrystals: constitutive equation and deformation mechanisms", J. Am. Ceram. Soc; 81 2761-2776 (1998).

8.-M. Jiménez-Melendo, A. Domínguez-Rodríguez, “High temperature mechanical characteristics of superplastic yttria-stabilized zirconia. An examination of the flow process", Acta mater. 48 3201-3210 (2000).

9.- B. E. Warren, "X-Ray Diffracction", p. 262, Dover Publications, Inc., New York, (USA) 1969.

10.-D. Gómez-García, C. Lorenzo-Martín, A. Muñoz-Bernabé and A. Domínguez-Rodríguez, "Correlation between yttrium segregation at the grain boundaries and the threshold stress for plasticity in yttria tetragonal zirconia polycrystals", Phil. Mag. A 83 [1] 93-108 (2003).

11.- D. Gómez-García, C. Lorenzo-Martín, A. Muñoz-Bernabé and A. Domínguez-Rodríguez, "Model of high-temperature plastic deformation of nanocrystalline materials: Application to yttria tetragonal zirconia"; Phys. Rev. B 67 144101-7 (2003).

12.- C. Lorenzo-Martín, D. Gómez-García, A. Gallardo-López, A. DomínguezRodríguez and R. Chaim, "An explanation of the high temperature creep of yttria tetragonal zirconia nanocrystals", Scripta mater. 50 1151-1155 (2004).

Recibido: 01.12.03

Aceptado: 23.11.04 